\title{
Nanostructure, solvation dynamics, and nanotemplating of plasmonically active SERS substrate in reverse vesicles
}

\author{
Ranajay Saha $\cdot$ Surajit Rakshit • \\ Dipanwita Majumdar - Achintya Singha • \\ Rajib Kumar Mitra $\cdot$ Samir Kumar Pal
}

Received: 27 September 2012/ Accepted: 9 March 2013

(C) Springer Science+Business Media Dordrecht 2013

\begin{abstract}
Reverse vesicles (RVs) are the organic counterparts to vesicles and are spherical containers in oils consisting of an oily core surrounded by reverse bilayers with water layers present in between. We present here a facile route for forming stable RV from nontoxic surfactants and oil components. The RV formation is characterized by dynamic light scattering and further confirmed by transmission electron microscopic (TEM) techniques. The water channels present in between the bilayers are found to be a potential template for inorganic nanoparticles' (NPs) synthesis. Both the UV-Vis absorption spectroscopy and the TEM study reveal successful formation of highly clustered silver NPs within the water layers of the RVs. X-ray powder diffraction analyzes the crystalline nature of the NPs. FTIR spectroscopy shows the signature of different kinds of water molecules in between the RV bilayers. The dynamical description of the templating water, dictating the controlled formation of the NPs in the RV, is well revealed in the picosecond-resolved solvation dynamics study of a hydrophilic fluorescence probe $2^{\prime}$-(4-hydroxyphenyl)-
\end{abstract}

R. Saha · S. Rakshit · R. K. Mitra · S. K. Pal ( $ه)$ Department of Chemical, Biological \& Macromolecular Sciences, S.N. Bose National Centre for Basic Sciences, Block JD, Sector III, Salt Lake, Kolkata 700098, India e-mail: skpal@bose.res.in

D. Majumdar · A. Singha

Department of Physics, Bose Institute, 93/1,

Acharya Prafulla Chandra Road, Kolkata 700 009, India 5-[5-(4-methylpiperazine-1-yl)-benzimidazo-2-yl-benzimidazole] (H258). The rotational anisotropy study successfully describes geometrical restriction of the probe molecule in the RV. Notably, this study provides the first proof-of-concept data for the ability of the $\mathrm{RV}$ to be a template of synthesizing metal NPs. The as-prepared NP clusters are evaluated to be potential surface-enhanced Raman scattering substrate in solution using crystal violet as a model analyte. The present study offers a new RV, which is a prospective nontoxic nanotemplate and is believed to contribute potentially in the emerging NP-vesicle hybrid assembly-based plasmonic applications.

Keywords Reverse vesicle $\cdot$ Nanotemplate . Picosecond-resolved solvation dynamics . Nanoparticle-vesicle hybrid · Nanoparticle clusters . SERS

\section{Introduction}

Self-assembled vesicular structures with a water-filled compartment enclosed by a thin shell of surfactants are of significant interest in many fields ranging from materials sciences to biophysics and nanomedicine (Discher and Eisenberg 2002; Guo and Szoka 2003; Sawant and Torchilin 2010). Much like the above "normal" vesicles in water, one can also find their counterparts in organic, nonpolar "oils," and these are 
termed "reverse" vesicles (RVs) (Tung et al. 2008; Kunieda et al. 1993; Li et al. 2010; Rangelov et al. 2004). With a nonpolar organic solvent being the continuous medium, amphiphilic molecules in RVs self-assemble in an opposite way with hydrophilic parts inside and hydrophobic parts outside (see Scheme 1). The study of RVs is of great fundamental interest since knowledge about molecular bilayers, which play an important role in living cells, can be obtained through a thoroughly new viewpoint. In this paper, we report a facile route for forming stable RVs from nontoxic surfactants in pharmaceutically viable nonpolar organic liquid isopropyl myristate (IPM).

RVs are rarer than normal vesicles, but one might imagine that they too could find numerous applications much like normal vesicles. The growing interest in functional vesicles has recently motivated research activities utilizing as building blocks metal and semiconductor nanoparticles (NP) with unique electronic, magnetic, and optical properties (Sanson et al. 2011; Al-Jamal et al. 2008; Fortuna et al. 2009; Song et al. 2011; Nyirő-Kósa et al. 2012; Solla-Gullón et al. 2010). NPs have been incorporated into polymersomes (Sanson et al. 2011) and liposomes (Al-Jamal et al. 2008; Chu et al. 2010) through noncovalent interactions. However, many of these techniques are subjected to limitations such as relatively low efficiency

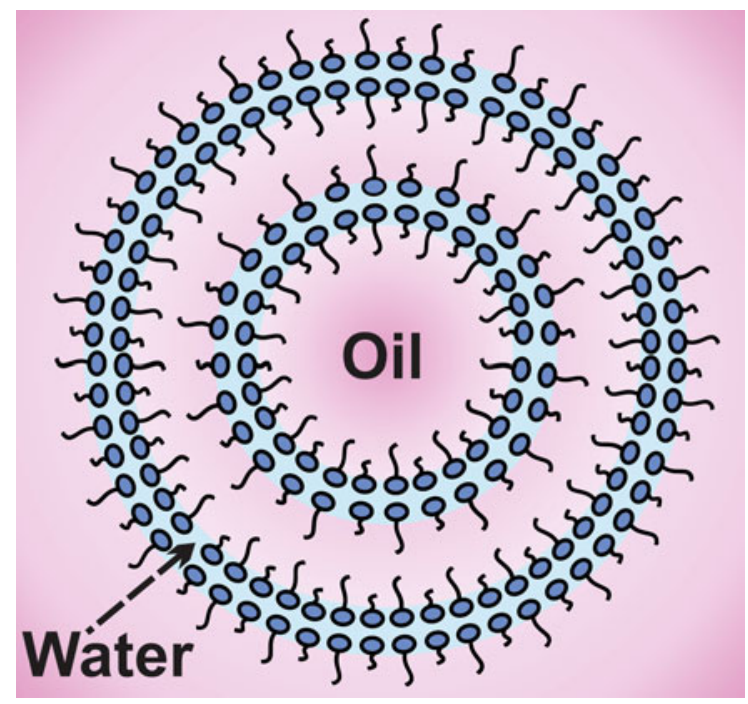

Scheme 1 Schematics of a typical structure of multilamellar reverse vesicle $(\mathrm{RV})$ in nonpolar oil medium. The present study shows that in the nonpolar oil isopropyl myristate, tween 80 can form RV in the presence of butyl lactate as cosurfactant and water as the polar medium and unsatisfactory yield. The continuous medium of the RV being oil, highly restricted two-dimensional water channels within its bilayers can efficiently trap inorganic metal ions ( $\mathrm{Li}$ et al. 2008) and provide a unique opportunity for highly productive synthesis of NP-vesicle hybrid assembly through in situ formation of highly clustered metal NPs in the vesicle template. Thus, one of the aims of the present study is to use RVs to be a suitable template for metal NP synthesis.

Remarkably, controlled growth of NPs from metal ions is essentially determined by the dynamics of the polar host environment. Thus, nanometer-sized water cores of reverse micelles (RMs) have been an attractive template for the production of NPs (Ethayaraja et al. 2006, 2007), in which the change in particle growth rate with $w_{0}(=$ [water]/[surfactant] $)$ values is found to be dictated by the rigidity of the water molecules in the nanoenvironment (Kitchens et al. 2003; Cason et al. 2001). Consequently, for the presence of only bound-type (slower) water molecules, NP synthesis is proved to be more difficult at $w_{0}<5$ (Smetana et al. 2007). On the other hand, with increased bulk-like behavior of water at higher $w_{0}$ $(>12)$, the isolated reaction environment is to some extent compromised, causing particle agglomeration and growth (Smetana et al. 2007). Hence, dynamical information of the nanotemplating water acting as the reaction media is essential for controlled formation of NPs in such confined spaces (Levinger 2002) and is probed here using the picosecond-resolved fluorescence spectroscopy of a hydrophilic solvation dye 2'-(4-hydroxyphenyl)-5-[5-(4-methylpiperazine-1-yl)benzimidazo-2-yl-benzimidazole] (H258) (Banerjee et al. 2007). To explore the geometrical restriction of the probe, rotational relaxation dynamics of the dye has been revealed using picosecond-resolved fluorescence anisotropy decay. Fourier transform infrared spectroscopy (FTIR) has been performed to determine the physicochemical characteristic of the entrapped water in the RV. Significantly, interparticle plasmonic coupling in an assembly produces collective properties different from those of the dispersed particles (Nie et al. 2010; Kinge et al. 2008; Cho et al. 2012). Therefore, RV assembly with high clustering of metal NPs could be a potential platform for surfaceenhanced Raman scattering (SERS) applications. To this end, we also study the SERS sensitivity of the asprepared NP clusters using crystal violet as a model analyte. We present here a novel RV composed 
of pharmaceutically acceptable ingredients tween 80/butyl lactate/IPM/water (Rowe et al. 2006; Saha et al. 2012). The RVs have been prepared by adding a measured amount of o/w microemulsion (ME, micelle) in excess of IPM. The details of the structural characterization including phase behavior of the ME were recently addressed from this group (Saha et al. 2012). The formation of RVs has been realized by dynamic light scattering (DLS) and transmission electron microscopy (TEM) techniques. The successful synthesis of metal NPs (silver) in the nanotemplate of $\mathrm{RVs}$ is confirmed by their characteristic surface plasmon resonance band and also by high resolution transmission electron microscopy (HRTEM). The crystalline nature of the NPs is characterized by X-ray powder diffraction (XRD). The present study offers a new RV, which is a nontoxic nanotemplate for inorganic NP synthesis. The formulation further offers a potential substrate made from NP-vesicle hybrid assembly for SERS-based molecular sensing for a trace amount of chemical analytes in solution.

\section{Experimental}

\section{Materials}

The following chemicals were obtained: isopropyl myristate (IPM, Sigma Aldrich), butyl lactate (Spectrochem), polyoxyethylenesorbitan monooleate (Tween 80; Sigma Aldrich), ammonium molybdate (SRL, analytical grade), silver nitrate $\left(\mathrm{AgNO}_{3}\right.$; Sigma Aldrich), sodium borohydride $\left(\mathrm{NaBH}_{4}\right.$, Sigma Aldrich), 2'-(4-hydroxyphenyl)-5-[5-(4-methylpiperazine-1-yl) -benzimidazo-2-yl-benzimidazole] (H258, Molecular Probes), and crystal violet (Sigma). All samples were of the highest commercially available purity and used without further purification. Double-distilled water was used throughout the experiments.

\section{Reverse vesicle $(\mathrm{RV})$ preparation}

For the preparation of the RVs, o/w ME comprising tween 80 , butyl lactate, and IPM, with the wt. fraction of water being $X_{\mathrm{w}}=0.37$, was prepared. The wt. ratio of tween 80 and butyl lactate was maintained at 1:1. The details of the ME preparation procedure can be seen from a recently published work from our group (Saha et al. 2012). Next, $13.3 \mu \mathrm{l}$ of the ME was added to $2 \mathrm{ml}$ of IPM. The solution was then vortexed for 15 min to get a transparent solution of RV.

\section{DLS study}

The hydrodynamic diameter of the RV was determined using the DLS instrument from Nano $\mathrm{S}$ Malvern instrument employing a $4 \mathrm{~mW} \mathrm{He}-\mathrm{Ne}$ laser $(\lambda=632.8 \mathrm{~nm})$ equipped with a thermostatted sample chamber. All the scattered photons are collected at $173^{\circ}$ scattering angle. The scattering intensity data are processed using the instrumental software to obtain the hydrodynamic diameter $\left(d_{\mathrm{H}}\right)$ and the size distribution of the scatterer in each sample. The instrument measures the time-dependent fluctuation in the intensity of light scattered from the particles in solution at a fixed scattering angle. The " $d_{\mathrm{H}}$ " of the nanodroplet is estimated from the intensity auto-correlation function of the time-dependent fluctuation in intensity and is defined as

$d_{\mathrm{H}}=\frac{k_{\mathrm{B}} T}{3 \pi \eta D}$

where $k_{\mathrm{B}}$ is the Boltzmann constant, $\eta$ is the viscosity, $D$ is the translational diffusion coefficient, and $T$ is the temperature. DLS experiments were done in triplicate with at least 15 runs per measurement.

\section{Particle synthesis in RV}

$200 \mathrm{mM}$ aqueous solution of $\mathrm{AgNO}_{3}$ was used as the aqueous phase to prepare ME with $X_{\mathrm{w}}=0.37 .13 .3 \mu \mathrm{l}$ of this ME was then added to $2 \mathrm{ml}$ of IPM and was allowed to stir for $15 \mathrm{~min}$ to get a transparent solution of RV containing silver ions. After that, $2.5 \mathrm{mg}$ of $\mathrm{NaBH}_{4}$ was added to the RV solutions and was allowed to stir overnight (12 h) to get RV containing silver NPs.

\section{Transmission electron microscopy (TEM)}

TEM measurements were performed on an FEI TecnaiTF-20 field-emission high resolution transmission electron microscope. $2.0 \mathrm{mM}$ ammonium molybdate solution was used as the aqueous phase of the RV for TEM imaging. Notably, molybdenum being a heavy metal is expected to deflect the electron beam 
producing a contrast in the TEM micrograph of the RVs and as such would clearly show the water layers of the RVs. For the TEM analysis, a drop of RV solution was deposited onto a carbon film supported by a copper grid and the solvent was then allowed to evaporate. The imaging of the RV was conducted at $120 \mathrm{kV}$ acceleration voltage of the microscope. For $\mathrm{RV}$ containing metal NPs, the acceleration voltage of the microscope was $200 \mathrm{kV}$ and imaging was done 20 days after its preparation.

\section{Fluorescence spectroscopic measurement}

Steady-state emission spectra were measured with a Jobin-Yvon Fluoromax-3 fluorimeter. Fluorescence transients were measured using a commercially available spectrophotometer (LifeSpec-ps) from Edinburgh Instrument, U.K. [excitation wavelength $375 \mathrm{~nm}$, $80 \mathrm{ps}$ instrument response function (IRF)] and fitted using F900 software provided by Edinburgh Instrument. Briefly, the observed fluorescence decay transients were fitted using a nonlinear least square fitting procedure to a function $\left(X(t)=\int_{0}^{t} E\left(t^{\prime}\right) R\left(t-t^{\prime}\right) \mathrm{d} t^{\prime}\right)$ comprising convolution of the IRF $(E(t))$ with a sum of exponentials $\left(R(t)=A+\sum_{i=1}^{N} B_{i} e^{-t / \tau_{i}}\right)$ with pre-exponential factors $\left(B_{i}\right)$, characteristic lifetimes $\left(\tau_{i}\right)$, and a background $(A)$. Relative concentration in a multiexponential decay is finally expressed as $a_{n}=\frac{B_{n}}{\sum_{i=1}^{N} B_{i}}$. The quality of the curve fitting is evaluated by reduced $\chi^{2}$ and residual data.

To construct time-resolved emission spectra (TRES), we followed the technique described in the reference (Lakowicz 1999). The time-dependent fluorescence Stokes shifts, as estimated from TRES, are used to construct the normalized spectral shift correlation function or the solvent correlation function, $C(t)$, defined as

$C(t)=\frac{v(t)-v(\infty)}{v(0)-v(\infty)}$

where $v(0), v(\mathrm{t})$, and $v(\infty)$ are the emission maxima (in $\mathrm{cm}^{-1}$ ) at time zero, $t$, and infinity, respectively. The $C(t)$ function represents the temporal response of the solvent relaxation process as occurring around the probe following its photo excitation and the associated change in the dipole moment. For anisotropy $(r(t))$ measurements, emission polarization was adjusted to be parallel or perpendicular to that of the excitation, and anisotropy is defined as (Lakowicz 1999)

$r(t)=\frac{I_{\mathrm{para}}(t)-G \times I_{\mathrm{per}}(t)}{I_{\mathrm{para}}(t)+2 \times G \times I_{\mathrm{per}}(t)}$

where $I_{\text {para }}(t)$ and $I_{\text {per }}(t)$ are the temporal emission intensities at parallel and perpendicular emission polarization, respectively, with respect to vertical excitation polarization. $G$, the grating factor, was determined following the longtime tail matching technique (Lakowicz 1999).

\section{Powder XRD measurement}

The structural properties of the synthesized silver NPs were analyzed by XRD with a PANalytical XPERT$\mathrm{PRO}$ diffractometer equipped with $\mathrm{Cu} \mathrm{K} \alpha$ radiation (at $40 \mathrm{~mA}, 45 \mathrm{kV}$ ). The NPs were ultracentrifuged and dried to get the XRD pattern.

UV-Vis/FTIR measurement

UV-Vis measurements were recorded on a Shimadzu UV2450 spectrophotometer. FTIR spectra were recorded on a JASCO FTIR-6300 spectrometer using $\mathrm{CaF}_{2}$ window.

\section{SERS measurement}

SERS measurements were performed in backscattering geometry using a LabRAM HR, JobinYvon, fitted with a Peltier-cooled charge-coupled device detector. An air-cooled argon ion laser with a wavelength of $488 \mathrm{~nm}$ was used as the excitation light source. The laser power was $17 \mathrm{~mW}$ at the sample and the integration time was $1 \mathrm{~s}$ with a double accumulation for each measurement. 2-4 $\mu \mathrm{l}$ of $560 \mu \mathrm{M} \mathrm{CV}$ ethanol solution was added in the substrate solutions, and SERS measurements were started after $5 \mathrm{~min}$ of incubation.

\section{Results and discussions}

To study the effect of adding the o/w ME (micelle, $X_{\mathrm{w}}=0.37$ ) in excess of nonpolar oil IPM, we conducted TEM. The TEM images in Fig. 1 show a number of spherical structures with distinct shells, much like the conventional micrographs of vesicles. Here, ammonium molybdate is used as the aqueous 
phase of the RV in order to deflect the electron beam, revealing a clear contrast between the peripheral and central areas of the reverse aggregates. Figure 1a shows three isolated unilamellar RVs having a prolate shape and the average sizes ranging from 20 to $30 \mathrm{~nm}$. The prolate shape of the RVs is also evident in Fig. 1c; however, the structure is distinctly multilayered with an average size of $400 \mathrm{~nm}$. Formation of prolate aggregates supports the hypothesis of the RVs in the present system (Mays et al. 1999). The multilamellar characteristic of the RVs is also evident in Fig. 1b; however, the shape is spherical with a diameter of $265 \mathrm{~nm}$. The individual bilayers in the multilamellar RVs are well separated from each other, indicating a more repulsive interaction (most likely enhanced undulation repulsion (McIntosh et al. 1989)) between the bilayers than it is found in the lamellar phase, in which the spacing of the bilayer is about the same as the bilayer thickness (Tardieu et al. 1973).

The formation of the RV is further confirmed by the DLS technique. Figure 1d shows the size distribution of the as-prepared reverse aggregates. The
DLS signals are found to be polydispersed with $\sim 70-460 \mathrm{~nm}$ of size distribution. The signature of a larger size distribution of the aggregates in these solutions compared to that in ME (ca $5 \mathrm{~nm}$ (Saha et al. 2012)) provides evidence for RV formation. The observed higher polydispersity in the size distribution indicates multilamellarity of the vesicles and is in agreement with the TEM data (Fig. 1) and the data published in the previous literature (Ushio et al. 1993). The RVs formed here are stable and do not revert back to the initial stage on prolonged standing as evidenced by the DLS study (data not shown).

To understand the physical nature of water molecules entrapped in the RV, we carried out FTIR measurements to determine the $\mathrm{O}-\mathrm{H}$ stretching frequency of water in the RVs. In order to avoid the contribution of $\mathrm{C}-\mathrm{H}$ stretching frequency, the observed spectra at the RV have been subtracted from the surfactant system without water, and the differential spectra have been analyzed. A representative result is depicted in Fig. 2a. It is known that different types of hydrogen-bonded water molecules exist in surfactant
Fig. 1 Representative TEM images of ammonium molybdate labeled reverse vesicles (RVs).

a Unilamellar RVs with prolate shape in the size range of $30 \mathrm{~nm}$. b A typical structure of multilamellar spherical RV is shown. Arrows indicate different spacing of the lamellar bilayers. c The 400-nm RV with ellipsoidal shape is distinctly multilayered. These RVs are formed when $13.3 \mu \mathrm{l}$ of the microemulsion is added to $2 \mathrm{ml}$ of isopropyl myristate. d The DLS signal of the as-prepared RVs
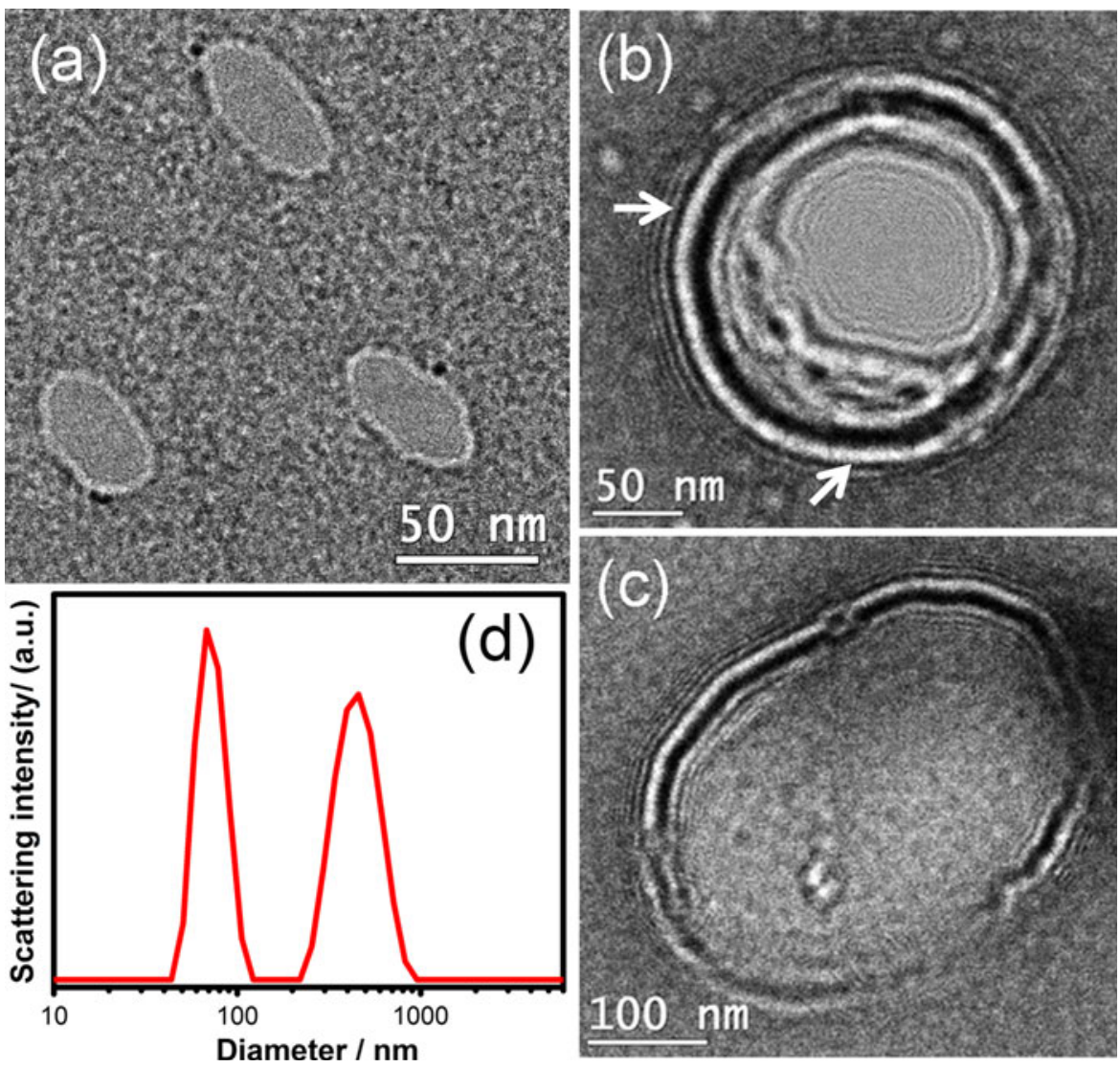
self-assemblies like RMs, which can broadly be classified into two major classes, namely headgroupbound and bulk-like water molecules (Mitra et al. 2008). The differential IR spectra obtained in the present study have been deconvoluted into two spectra peaking at $\sim 3,425$ and $\sim 3,390 \mathrm{~cm}^{-1}$, corresponding to the $\mathrm{O}-\mathrm{H}$ stretching frequency of the headgroupbound and bulk-like water, respectively. To probe the dynamical states of such water confined in the RV, polar solvation dye $\mathrm{H} 258$ has been used. Figure $2 \mathrm{~b}$ shows the steady-state excitation and emission spectra of $\mathrm{H} 258$ in the buffer and RV. In the buffer, the excitation and emission peaks of $\mathrm{H} 258$ are at 366 and $500 \mathrm{~nm}$, respectively. However, these are significantly blueshifted to 355 and $468 \mathrm{~nm}$ in the RV. It may be recalled that in hydrophobic environments, H258 shows blueshifted absorption and emission peak (Banerjee et al. 2007). Accordingly, the observed blueshift in the excitation and emission peaks of $\mathrm{H} 258$ in the $\mathrm{RV}$ is consistent with the presence of a considerable fraction of $\mathrm{H} 258$ molecules at the surfactant-water interface of the RV where the microenvironment polarity is expected to be lower compared to bulk water.

Figure 3a shows the decay transients of $\mathrm{H} 258$ in the $\mathrm{RV}$ s at three selected wavelengths of 430 (at the blue end of the spectrum), 470 (at the peak position), and 530 (at the red end of the spectrum) nm. The transient at $430 \mathrm{~nm}$ can be fitted tri-exponentially with time components of $0.09 \mathrm{~ns}(50 \%), 0.98 \mathrm{~ns}$ (31\%), and $2.97 \mathrm{~ns}(19 \%)$. For the extreme red wavelength $(530 \mathrm{~nm})$, a distinct rise component of $0.09 \mathrm{~ns}$ is obtained along with the decay components of 2.05 and $4.35 \mathrm{~ns}$. The presence of faster decay components at the blue end and a rise component at the red wavelength is indicative of solvation (Saha et al. 2011) of the probe in the RV system within the experimental time window.

Using the decay transients at different wavelengths, we constructed time-resolved emission spectra (TRES) of the probe in the RV. Figure $3 b$ shows the representative TRES for $\mathrm{H} 258$ in the RV, wherein a significant dynamic fluorescence Stokes shift of $1,300 \mathrm{~cm}^{-1}$ in $9 \mathrm{~ns}$ is observed. The solvent correlation function, $C(t)$, obtained is fitted bi-exponentially (Fig. 3c) based on the core-shell model (Piletic et al. 2006) with time components of $2.26 \mathrm{~ns}(45 \%)$ and $0.12 \mathrm{~ns}(55 \%)$. It can be noted that both these components are slower than the subpicosecond solvation time scale reported
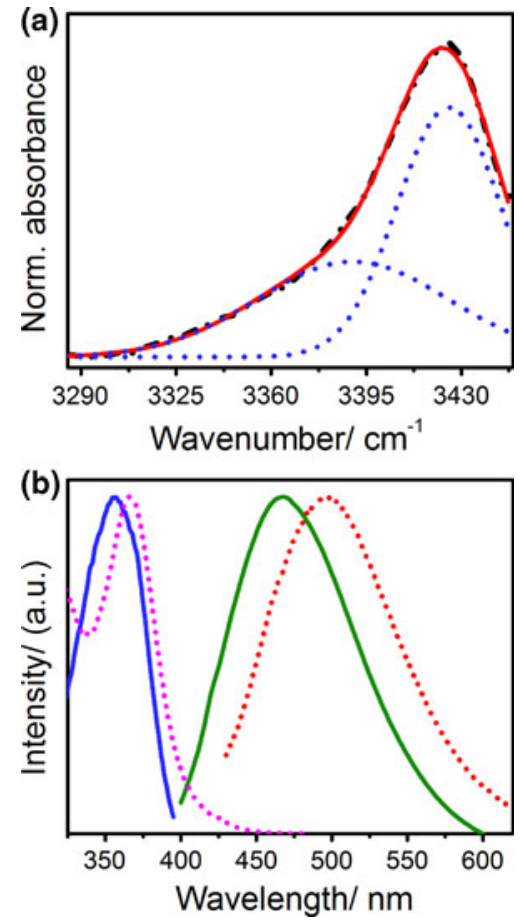

Fig. 2 a FTIR spectra of reverse vesicle (RV). The black (dash dotted) curve is the experimental one, the red (solid) curve is the overall fitted data, and the blue (dotted) lines are the deconvoluted curves. b Excitation and emission spectra of $\mathrm{H} 258$ in RV (solid lines). The dotted lines are the corresponding spectra in buffer. (Color figure online)

for bulk water (Jimenez et al. 1994). As has been inferred from steady-state measurements, the observed solvation dynamics appears to be due exclusively to the $\mathrm{H} 258$ molecules residing at the surfactant-water interface of the RVs. The observed average solvation time $\left(\left\langle\tau_{s}\right\rangle=a_{1} \tau_{1}+a_{2} \tau_{2}\right)$ of $1.08 \mathrm{~ns}$ is comparable to that of $\mathrm{H} 258$ molecules located in the hydration shell at the interface of the nanotemplating AOT RM (Banerjee et al. 2007) and essentially corresponds to the dynamical exchange between headgroup-bound and interfacially bound water. Successful probing of interfacial water dynamics is evident in the restricted rotation of the probe in the RV. The rotational anisotropy of the dye in the RV is shown in the inset of Fig. 3c. It could be noted that $\mathrm{H} 258$ in bulk water shows the rotational lifetime, $\tau_{\mathrm{r}}$, of $500 \mathrm{ps}$ (Banerjee and $\mathrm{Pal}$ 2006), indicative of the free rotational motion. However, in the RV, the rotational anisotropy of the probe is significantly slower and even does not complete within the time window of 6 ns (inset of Fig. 3b). This shows that the dye is experiencing higher 


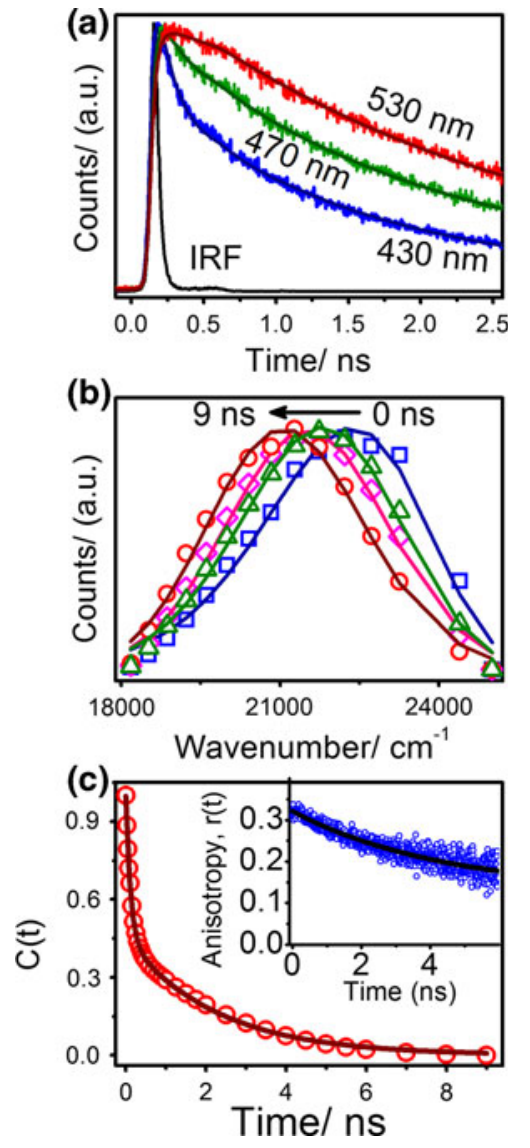

Fig. 3 a Fluorescence decay transients of H258 in reverse vesicle (RV). b Time-resolved emission spectra (TRES) of $\mathrm{H} 258$ in RV. c Solvent correlation function, $C(t)$, of H258 in RV. The solid lines denote the best fit to the biexponential decay. Inset shows the picosecond-resolved fluorescence anisotropy decay of $\mathrm{H} 258$ in $\mathrm{RV}$

microviscosity in the RVs in comparison to that in bulk water and illustrates the residence of the probe in the interfacial layer of the RV.

The water nanochannels present in between the bilayers of the RV show dynamic behavior typical of a nanotemplating RM (discussed earlier) and can trap metal ions like $\mathrm{MoO}_{4}^{-2}$ quite efficiently (Fig. 1). Thus, the RV water layers are expected to be a potential template for inorganic metal NP synthesis. The formation of silver NPs in the RV template is realized in the HRTEM and UV-Vis absorption studies. Figure $4 \mathrm{a}$ displays the absorption spectrum of the as-obtained silver NPs exhibiting the characteristic surface plasmon bands at $400 \mathrm{~nm}$ (Kemp et al. 2009). The in situ formation and inclusion of silver NPs between lamellae are shown in the TEM images of
Fig. $4 \mathrm{~b}, \mathrm{~d}$, and $\mathrm{f}$. The HRTEM image in Fig. 4c shows an isolated spherical silver NP of $9.10 \mathrm{~nm}$ size, with clear lattice fringes of interplane distance of $0.20 \mathrm{~nm}$ corresponding to the (200) lattice space of metallic silver (Behrens et al. 2004). The fringe pattern shows the high quality of the nanocrystals. The polydispersed size distribution of the metallic silver (ranging between 4.3 and $13 \mathrm{~nm}$ ), as revealed in Fig. $4 \mathrm{e}$, is quite consistent with variable interlamellar distance of the RVs (Fig. 1b). The existence of distinct isolated clusters of silver NPs found in the size range of the RVs suggests that after particle synthesis, the overall aggregate structure of the RV is retained. According to the colloidal particle nucleation and growth model proposed by La Mer and Dinegar, particle nucleation occurs when the ion concentration reaches supersaturation concentrations (LaMer and Dinegar 1950). This condition is likely to be met when metallic salt (like $\mathrm{Ag}^{+}$, here) is already present in the aqueous phase during the formation of the RV as has been adopted here. In this condition, addition of an external reducing agent (like $\mathrm{NaBH}_{4}$ ) may lead to the simultaneous nucleation of NPs at many sites of the vesicle walls, and many particles can grow within the bilayers. Importantly, the gradual diffusion of reductant along the RV bilayer is believed to be responsible for particle growth in a controlled manner forming smaller size $(4.3-13 \mathrm{~nm}) \mathrm{NPs}$. In fact, the finite vesicular volume places a restriction on the number of metal ions available for crystal nucleation and growth, resulting in particles significantly smaller than those formed in the extravesicular phase and additionally can avoid particle aggregation (Meldrum et al. 1993). Significantly, the dispersing media of the RV being oil, particle synthesis can occur only in the vesicle water layers, and the possibility of bulk synthesis (extravesicular synthesis) as can occur in normal vesicles is totally eliminated. In this regard, the clustering of the NPs (Fig. 4b, f) strongly suggests templated particle formation and growth, indicating complete prevention of extravesicular particle synthesis. Consequently, the prevention of such extravesicular particle synthesis makes RVs much more attractive than unilamellar vesicles or even classical multilamellar vesicles for particle synthesis. Notably, formation of classical vesicles requires sonication or mechanical stirring. In such systems, vesicle components are mixed with an excess solution of metallic salt. Consequently, not just does the exact internal 
Fig. 4 a UV-Vis absorption spectra of silver nanoparticles (NPs) obtained in the nanotemplate of reverse vesicles (RVs). b TEM micrograph of silver NPs' loaded RVs. c HRTEM image of a spherical silver NP with clear lattice fringes. d Silver NPs produced in RVs of different sizes. e HRTEM image of silver NPs produced at the RV oil interface with different particle size. f Isolated silver NPs, highly clustered in various RVs
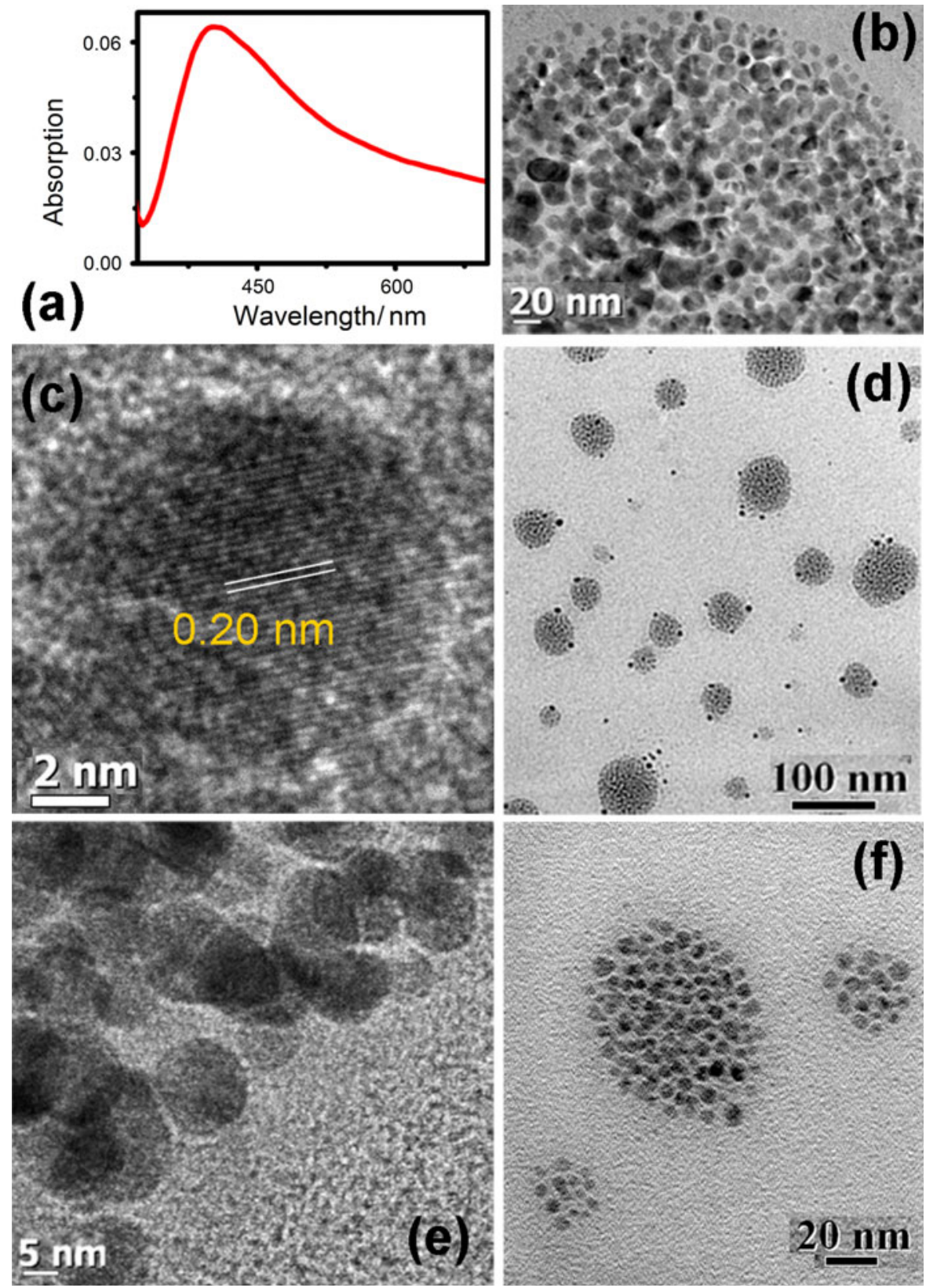

concentration of the metal remain unknown, but the extravesicular particle synthesis cannot be avoided (Tricot and Fendler 1986), unless a separation step [either by column chromatography (Mann et al. 1986) or dialysis (Meldrum et al. 1993)] is performed to remove unencapsulated metal ions. Since the continuous medium of the RV is oil, polar metal ions are localized only in the aqueous layer of the RVs, which avoids any separation step as required for classical vesicle-directed particle synthesis. Thus, the RVdirected particle synthesis is more efficient and is economically advantageous over the classical vesiclemediated synthetic procedure.

The crystal structure of the synthesized silver NPs is analyzed by powder XRD measurements. Figure 5 shows a typical XRD pattern of the as-prepared product. The discernible peaks can be indexed to (111), (200), (220), (311), and (222) crystal planes of 


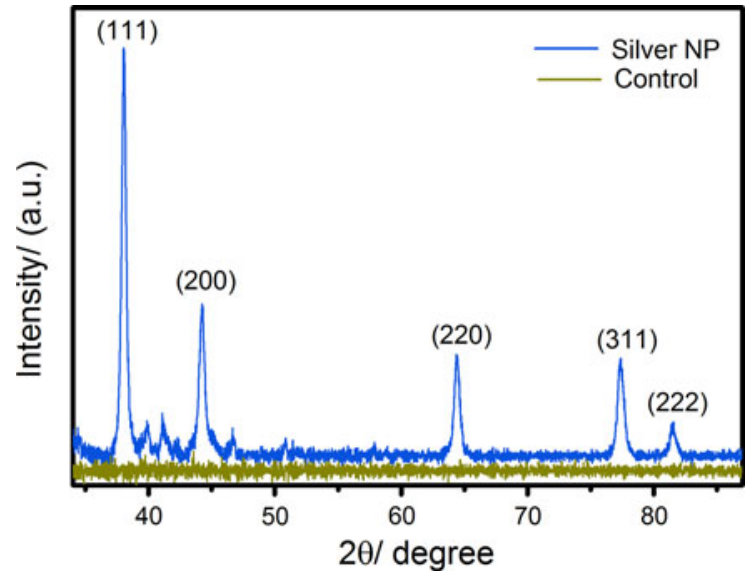

Fig. 5 XRD pattern of silver nanoparticles synthesized from reverse vesicles (RVs). The control set denotes the XRD pattern of the RVs

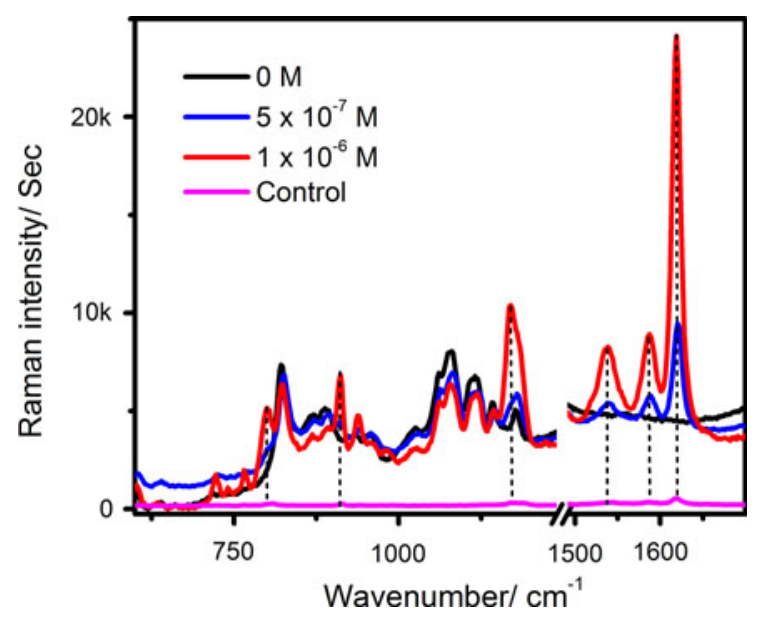

Fig. 6 SERS spectra of crystal violet at various concentrations for the silver nanoparticle cluster in reverse vesicles (RVs). The control set denotes the bulk Raman spectra of $1.9 \times 10^{-4} \mathrm{M}$ of crystal violet in water. Vertical lines mark positions of some characteristic vibrational bands of crystal violet

face-centered cubic structure of metallic silver (Chen et al. 2008). The XRD pattern thus clearly illustrates that the silver NPs formed in this present synthesis are crystalline in nature. According to the Debye-Scherrer equation, the diameter of the silver NPs is estimated to be ca $14.5 \mathrm{~nm}$, which is relatively close to the size range of the silver NPs as shown in the TEM images.

The templated synthesis of highly clustered metal NPs in the RV template offers a unique opportunity to use these NP-vesicle hybrid assemblies in various plasmonic applications. Interestingly, electromagnetic (EM) field is intensively localized into a nanoscale junction of metal NPs to create significant field enhancement, referred to as "hotspots" (Kreibig and Vollmer 1995; Zhang et al. 2011; Muniz-Miranda et al. 2011). This huge EM field localization resonant coupling with the surface plasmon of metal NPs is crucial for various SERS applications like biosensing (Wang et al. 2010), label-free immunoassays (Schultz et al. 2000), etc. Thus, NP clusters of nobel metals are attractive for SERS sensors due to the strong EM field enhancements arising at the interparticle junctions upon interaction with visible radiation (Gellini et al. 2008). To get insight into the SERS detection performance of the highly dense cluster of silver NPs as revealed in Fig. 4b, d, and f, we used crystal violet (CV) as a model analyte. Figure 6 shows the SERS spectra of $\mathrm{CV}$ at various concentrations adsorbed on the silver NP clusters, measured with an excitation wavelength of $488 \mathrm{~nm}$. The SERS spectra reveal the characteristic peaks of CV (even in trace amounts) with very high intensity at most of the earlier reported frequencies (Cañamares et al. 2008). Considerable enhancement of the SERS signal at 800, 912, 1170, 1537,1587 , and $1619 \mathrm{~cm}^{-1}$ with respect to the bulk Raman spectra of CV can be observed within the scale limit of the diagram. The SERS enhancement factor (EF) estimated from the signal intensities of the ring $\mathrm{C}-\mathrm{C}$ stretching mode at $1,619 \mathrm{~cm}^{-1}$ with respect to the bulk Raman spectra of the molecule $(193 \mu \mathrm{M})$ is in the range of ca $10^{4}$. This superior enhancement of the Raman signal and ultrasensitivity to the analyte is ascribed to the high clustering of the silver NPs in the confinement of the RV, which laterally confine the surface plasmons in a very small volume that can efficiently couple with the incident laser (Cho et al. 2012). It is important to note that in EF estimation, it was assumed that all CV molecules adsorb with the same efficiency on the silver NP surfaces and/or contribute to the measured signal. However, it is more likely that a small fraction of the molecules will contribute to the observed intensities as $\mathrm{CV}$ ethanol is fairly soluble in IPM. It is again important to note that $\mathrm{CV}$ does not exhibit a significant absorption band around $488 \mathrm{~nm}$, which excludes the possibility of any resonance Raman effects for the excitation laser employed in our study. Further enhancement of the SERS effect can be achieved by improving the cluster density of the substrate solution, employing probe molecules with resonance effects, and by optimizing the laser wavelength employed in the measurements. 


\section{Conclusion}

To summarize, we prepared a new RV from edible surfactant and oil components. The formation of the RVs has been confirmed by the DLS and TEM studies. The structures are found to be stable on aging and are of different lamellarity, with diameters ranging from $\sim 70$ to $460 \mathrm{~nm}$. FTIR spectroscopy reveals the signature of different kinds of water molecules in between the RV bilayers. Micropolarity of the water layers is successfully probed by the solvatochromic dye $\mathrm{H} 258$. Both steady-state excitation and emission spectra show the dye to reside in the surfactant-water interface of the RVs. The significant rotational hindrance of the dye in the time-resolved fluorescence anisotropy study further confirms its presence at the interface. Picosecond time-resolved fluorescence spectroscopy suggests the dynamics of water to be significantly slower compared to bulk water and very much similar to the nanotemplating water of AOT RMs. Accordingly, the RVs formed are evaluated as a potential template in controlled growth of highly crystalline silver NPs (nanoparticles) with high efficiency and substantial productivity. Powder XRD analyzes the face-centered cubic nature of the silver NPs. The TEM study shows high clustering of the NPs in the confinement of the RVs. These NP clusters are evaluated as potential SERS substrates in solution using $\mathrm{CV}$ as a reporter molecule. The high quality of the spectra obtained for the model compound demonstrates the efficiency of the prepared substrate for SERS enhancement and its potential as a nontoxic SERS detection probe for chemical and biological analysis in situ. Notably, this study provides the first proof-of-concept data for the ability of the RV to be a template of synthesizing metal NPs. Based on the potential of the RV for biomimetic nanomaterials' preparation demonstrated here, we expect that novel applications based on self-assembled NP-vesicle hybrid assembly would evolve.

Acknowledgments SR thanks the Council of Scientific and Industrial Research (CSIR) for a fellowship. We thank the Department of Science and Technology (DST), India, for the financial support (No. DST/TM/SERI/2k11/103).

\section{References}

Al-Jamal WT, Al-Jamal KT, Tian B, Lacerda L, Bomans PH, Frederik PM, Kostarelos K (2008) Lipid-quantum dot bilayer vesicles enhance tumor cell uptake and retention in vitro and in vivo. ACS Nano 2(3):408-418. doi: $10.1021 / \mathrm{nn} 700176 \mathrm{a}$

Banerjee D, Pal SK (2006) Ultrafast charge transfer and solvation of DNA minor groove binder: Hoechst 33258 in restricted environments. Chem Phys Lett 432(1-3):257262. doi:10.1016/j.cplett.2006.10.018

Banerjee D, Sinha SS, Pal SK (2007) Interplay between hydration and electrostatic attraction in ligand binding: direct observation of hydration barrier at reverse micellar interface. J Phys Chem B 111(51):14239-14243. doi: 10.1021/jp076392e

Behrens S, Wu J, Habicht W, Unger E (2004) Silver nanoparticle and nanowire formation by microtubule templates. Chem Mater 16(16):3085-3090. doi:10.1021/cm 049462s

Cañamares MV, Chenal C, Birke RL, Lombardi JR (2008) DFT, SERS, and single-molecule SERS of crystal violet. J Phys Chem C 112(51):20295-20300. doi:10.1021/jp807807j

Cason JP, Miller ME, Thompson JB, Roberts CB (2001) Solvent effects on copper nanoparticle growth behavior in AOT Reverse micelle systems. J Phys Chem B 105(12):22972302. doi:10.1021/jp002127g

Chen M, Ding W, Kong Y, Diao G (2008) Conversion of the surface property of oleic acid stabilized silver nanoparticles from hydrophobic to hydrophilic based on host-guest binding interaction. Langmuir 24(7):3471-3478. doi: 10.1021/la704020j

Cho WJ, Kim Y, Kim JK (2012) Ultrahigh-density array of silver nanoclusters for SERS substrate with high sensitivity and excellent reproducibility. ACS Nano 6(1):249-255. doi: $10.1021 / \mathrm{nn} 2035236$

Chu M, Zhuo S, Xu J, Sheng Q, Hou S, Wang R (2010) Liposome-coated quantum dots targeting the sentinel lymph node. J Nanopart Res 12(1):187-197. doi:10.1007/s11051009-9593-2

Discher DE, Eisenberg A (2002) Polymer vesicles. Science 297(5583):967-973. doi:10.1126/science.1074972

Ethayaraja M, Dutta K, Bandyopadhyaya R (2006) Mechanism of nanoparticle formation in self-assembled colloidal templates: population balance model and Monte Carlo simulation. J Phys Chem B 110(33):16471-16481. doi: 10.1021/jp0623645

Ethayaraja M, Dutta K, Muthukumaran D, Bandyopadhyaya R (2007) Nanoparticle formation in water-in-oil microemulsions: experiments, mechanism, and Monte Carlo simulation. Langmuir 23(6):3418-3423. doi:10.1021/la062896c

Fortuna S, Colard CAL, Troisi A, Bon SAF (2009) Packing patterns of silica nanoparticles on surfaces of armored polystyrene latex particles. Langmuir 25(21):12399-12403. doi: 10.1021/la9010289

Gellini C, Muniz-Miranda M, Innocenti M, Carla F, Loglio F, Foresti ML, Salvi PR (2008) Nanopatterned Ag substrates for SERS spectroscopy. Phys Chem Chem Phys 10(31): 4555-4558. doi:10.1039/B807663D

Guo X, Szoka FC (2003) Chemical approaches to triggerable lipid vesicles for drug and gene delivery. Acc Chem Res 36(5):335-341. doi:10.1021/ar9703241

Jimenez R, Fleming GR, Kumar PV, Maroncelli M (1994) Femtosecond solvation dynamics of water. Nature 369(6480): 471-473. doi:10.1038/369471a0 
Kemp MM, Kumar A, Mousa S, Park T-J, Ajayan P, Kubotera N, Mousa SA, Linhardt RJ (2009) Synthesis of gold and silver nanoparticles stabilized with glycosaminoglycans having distinctive biological activities. Biomacromolecules 10(3):589-595. doi:10.1021/bm801266t

Kinge S, Crego-Calama M, Reinhoudt DN (2008) Self-assembling nanoparticles at surfaces and interfaces. ChemPhysChem 9(1):20-42. doi:10.1002/cphc.200700475

Kitchens CL, McLeod MC, Roberts CB (2003) Solvent effects on the growth and steric stabilization of copper metallic nanoparticles in AOT reverse micelle systems. J Phys Chem B 107(41):11331-11338. doi:10.1021/jp0354090

Kreibig U, Vollmer M (1995) Optical properties of metal clusters. Springer, Berlin

Kunieda H, Nakamura K, Olsson U, Lindman B (1993) Spontaneous formation of reverse vesicles. J Phys Chem 97(37):9525-9531. doi:10.1021/j100139a043

Lakowicz JR (1999) Principles of fluorescence spectroscopy. Kluwer Academic/Plenum, New York

LaMer VK, Dinegar RH (1950) Theory, production and mechanism of formation of monodispersed hydrosols. J Am Chem Soc 72(11):4847-4854. doi:10.1021/ja01167a001

Levinger NE (2002) Water in confinement. Science 298(5599):1722-1723. doi:10.1126/science.1079322

Li H, Hao J, Wu Z (2008) Phase behavior and properties of reverse vesicles in salt-free catanionic surfactant mixtures. J Phys Chem B 112(12):3705-3710. doi:10.1021/jp7112329

Li H, Xin X, Kalwarczyk T, Kalwarczyk E, Niton P, Hołyst R, Hao J (2010) Reverse vesicles from a salt-free catanionic surfactant system: a confocal fluorescence microscopy study. Langmuir 26(19):15210-15218. doi:10.1021/la1029068

Mann S, Hannington JP, Williams RJP (1986) Phospholipid vesicles as a model system for biomineralization. Nature 324(6097):565-567. doi:10.1038/324565a0

Mays H, Almgren M, Dedinaite A, Claesson PM (1999) Spontaneous formation of reverse vesicles with soybean phosphatidyl ethanolamine in mixture with triglyceride and some water. Langmuir 15(23):8072-8079. doi:10.1021/la990433j

McIntosh TJ, Magid AD, Simon SA (1989) Repulsive interactions between uncharged bilayers-hydration and fluctuation pressures for monoglycerides. Biophys J 55(5):897904. doi:10.1016/S0006-3495(89)82888-7

Meldrum FC, Heywood BR, Mann S (1993) Influence of membrane composition on the intravesicular precipitation of nanophase gold particles. J Colloid Interface Sci 161(1):66-71. doi:10.1006/jcis.1993.1442

Mitra RK, Sinha SS, Verma PK, Pal SK (2008) Modulation of dynamics and reactivity of water in reverse micelles of mixed surfactants. J Phys Chem B 112(41):12946-12953. doi:10.1021/jp803585q

Muniz-Miranda M, Gellini C, Salvi PR, Innocenti M, Pagliai M, Schettino V (2011) Fabrication of nanostructured silver substrates for surface-enhanced Raman spectroscopy. J Nanopart Res 13(11):5863-5871. doi:10.1007/s11051011-0493-x

Nie Z, Petukhova A, Kumacheva E (2010) Properties and emerging applications of self-assembled structures made from inorganic nanoparticles. Nat Nanotechnol 5(1):15-25. doi:10.1038/nnano.2009.453

Nyirő-Kósa I, Rečnik A, Pósfai M (2012) Novel methods for the synthesis of magnetite nanoparticles with special morphologies and textured assemblages. J Nanopart Res 14(10):1-10. doi:10.1007/s11051-012-1150-8

Piletic IR, Moilanen DE, Spry DB, Levinger NE, Fayer MD (2006) Testing the core/shell model of nanoconfined water in reverse micelles using linear and nonlinear IR spectroscopy. J Phys Chem A 110(15):4985-4999. doi: $10.1021 /$ jp061065c

Rangelov S, Almgren M, Edwards K, Tsvetanov C (2004) Formation of normal and reverse bilayer structures by self-assembly of nonionic block copolymers bearing lipid-mimetic units. J Phys Chem B 108(23):7542-7552. doi:10.1021/jp0304576

Rowe RC, Sheskey PJ, Owen SC (eds) (2006) Handbook of pharmaceutical excipients, 5th edn. Pharmaceutical Press and American Pharmacists Association, London

Saha R, Verma PK, Mitra RK, Pal SK (2011) Structural and dynamical characterization of unilamellar AOT vesicles in aqueous solutions and their efficacy as potential drug delivery vehicle. Colloids Surf B 88(1):345-353. doi: 10.1016/j.colsurfb.2011.07.012

Saha R, Rakshit S, Mitra RK, Pal SK (2012) Microstructure, morphology, and ultrafast dynamics of a novel edible microemulsion. Langmuir 28(22):8309-8317. doi:10.1021/la 3012124

Sanson C, Diou O, Thévenot J, Ibarboure E, Soum A, Brûlet A, Miraux S, Thiaudière E, Tan S, Brisson A, Dupuis V, Sandre O, Sb Lecommandoux (2011) Doxorubicin loaded magnetic polymersomes: theranostic nanocarriers for MR imaging and magneto-chemotherapy. ACS Nano 5(2): 1122-1140. doi:10.1021/nn102762f

Sawant RR, Torchilin VP (2010) Liposomes as 'smart' pharmaceutical nanocarriers. Soft Matter 6(17):4026-4044. doi:10.1039/B923535N

Schultz S, Smith DR, Mock JJ, Schultz DA (2000) Single-target molecule detection with nonbleaching multicolor optical immunolabels. Proc Natl Acad Sci USA 97(3):996-1001. doi:10.1073/pnas.97.3.996

Smetana AB, Wang JS, Boeckl J, Brown GJ, Wai CM (2007) Fine-tuning size of gold nanoparticles by cooling during reverse micelle synthesis. Langmuir 23(21):10429-10432. doi: $10.1021 / 1 a 701229 q$

Solla-Gullón J, Gómez E, Vallés E, Aldaz A, Feliu J (2010) Synthesis and structural, magnetic and electrochemical characterization of PtCo nanoparticles prepared by waterin-oil microemulsion. J Nanopart Res 12(4):1149-1159. doi:10.1007/s11051-009-9680-4

Song J, Cheng L, Liu A, Yin J, Kuang M, Duan H (2011) Plasmonic vesicles of amphiphilic gold nanocrystals: selfassembly and external-stimuli-triggered destruction. J Am Chem Soc 133(28):10760-10763. doi:10.1021/ja204387w

Tardieu A, Luzzati V, Reman FC (1973) Structure and polymorphism of the hydrocarbon chains of lipids: a study of lecithin-water phases. J Mol Biol 75(4):711-733. doi: 10.1016/0022-2836(73)90303-3

Tricot YM, Fendler JH (1986) In situ generated colloidal semiconductor cadmium sulfide particles in dihexadecyl phosphate vesicles: quantum size and symmetry effects. J Phys Chem 90(15):3369-3374. doi:10.1021/j100406a013

Tung S-H, Lee H-Y, Raghavan SR (2008) A facile route for creating "reverse" vesicles: insights into "reverse" self-assembly in organic liquids. J Am Chem Soc 130(27): 8813-8817. doi:10.1021/ja801895n 
Ushio N, Solans C, Azemer NHK (1993) Formation and stability or reverse vesicles in a sucrose alkanoate system. J Jpn Oil Chem Soc (Yukagaku) 42(11):915-922. doi:10.5650/jos 1956.42.915

Wang Y, Seebald JL, Szeto DP, Irudayaraj J (2010) Biocompatibility and biodistribution of surface-enhanced raman scattering nanoprobes in zebrafish embryos: in vivo and multiplex imaging. ACS Nano 4(7):4039-4053. doi: 10.1021/nn100351h

Zhang D-F, Zhang Q, Niu L-Y, Jiang L, Yin P-G, Guo L (2011) Self-assembly of gold nanoparticles into chain-like structures and their optical properties. J Nanopart Res 13(9): 3923-3928. doi:10.1007/s11051-011-0312-4 